\title{
Rotation Period of Venus estimated from Venus Express VIRTIS images and Magellan altimetry
}

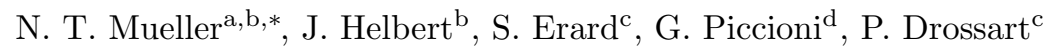 \\ ${ }^{a}$ Institut für Planetologie, Westfälische Wilhelms-Universität Münster, Wilhelm-Klemm-Str. 10, \\ 48149 Münster, Germany \\ ${ }^{b}$ Institute of Planetary Research, DLR, Rutherfordstr. 2, 12489 Berlin , Germany \\ ${ }^{c}$ LESIA, Observatoire de Paris, 61 Avenue Observatoire, F-75014 Paris, France. \\ ${ }^{d}$ IASF-INAF, via del fosso del cavaliere 100, Rome I-00133, Italy
}

\section{Abstract}

The $1.02 \mu \mathrm{m}$ wavelength thermal emission of the night side of Venus is strongly anticorrelated to the elevation of the surface. The VIRTIS instrument on Venus Express has mapped this emission and therefore gives evidence for the orientation of Venus between 2006 and 2008. The Magellan mission provided a global altimetry data set recorded between 1990 and 1992. Comparison of these two data sets reveals a deviation in longitude indicating that the rotation of the planet is not fully described by the orientation model recommended by the IAU. This deviation is sufficiently large to affect estimates of surface emissivity from infrared imaging. A revised period of rotation of Venus of $243.023 \pm 0.002$ days aligns the two data sets. This period of rotation agrees with pre-Magellan estimates but is significantly different from the commonly accepted value of $243.0185 \pm 0.0001$ days estimated from Magellan radar images. It is possible that this discrepancy stems from a length of day variation with the value of $243.023 \pm 0.002$ days representing the average of the rotation period over 16 years.

Keywords: Venus, Rotational dynamics, Infrared observations

\section{1. Introduction}

12 Venus Express is the first spacecraft orbiting Venus since the end of the Magellan 13 Mission in 1994. By comparing the appropriate data sets from these mission, we can

\footnotetext{
* Corresponding author

Email address: nils.mueller@dlr.de (N. T. Mueller)

Preprint submitted to Icarus
} 
14 estimate the rotation of the planet accumulated over 16 years and thus the mean rotation 15 period.

The atmosphere of Venus is optically thick in visible and infrared wavelengths and 17 the first tenable estimates of the slow retrograde rotation of the surface were derived 18 from Earth based radar observations (e.g. Victor and Stevens, 1961; Pettengill et al., 19 1962; Goldstein and Carpenter, 1963). The early estimates were based on the spectral

44 (Konopliv et al., 1999). 
The cited rotation period determinations from surface feature tracking are based on imaging of radar surface reflectivity. By contrast, several instruments on Venus Express can track surface features by observing near infrared atmospheric windows that transmit some of the thermal emission from the surface. The observations of thermal emission used here are from the first band of the infrared channel of the visible and thermal imaging spectrometer (VIRTIS), approximately at $1.02 \mu \mathrm{m}$ wavelength (Coradini et al., 1998; Drossart et al., 2007). Due to the extreme greenhouse climate and thick atmosphere the surface temperature can assumed to be a function of surface elevation. Accordingly, thermal emission is strongly anticorrelated to radar altimetry (Lecacheux et al., 1993).

Thermal emission is also influenced by the surface emissivity, which is of geological interest as it yields information about the chemistry and mineralogy of the surface (Helbert et al., 2008; Hashimoto et al., 2008; Arnold et al., 2008; Mueller et al., 2008; Smrekar et al., 2010; Haus and Arnold, 2010). The surface emissivity can be derived, when thermal emission imaging data can be combined with sufficiently accurate radar altimetry (Hashimoto and Sugita, 2003), e.g. the Magellan global topography data record (GTDR) (Ford and Pettengill, 1992; Rappaport et al., 1999).

The VIRTIS data in combination with GTDR altimetry have revealed increased emissivity at several volcanoes (Mueller et al., 2008), which is interpreted as resulting from fresh, relatively unweathered lava flows Smrekar et al. (2010). In areas with less obvious emissivity anomalies, closer inspection shows that westward slopes have a tendency to appear brighter than predicted by GTDR altimetry. A misalignment between the coordinate system used and the actual orientation of the planet qualitatively fits the observed bias. Smrekar et al. (2010) applied a shift of $-0.15^{\circ}$ in longitude to improve the emissivity maps from the work of Mueller et al. (2008). This shift minimizes the derived emissivity variation, however it does not strongly affect the most obvious emissivity anomalies.

The retrieval of surface emissivity is based on the prediction of thermal emission from altimetry. Alternatively, the VIRTIS thermal emission data can be used to estimate surface topography, but this estimate is locally biased by the unknown surface emissivity. This study is conducted using the topography derived from VIRTIS because it involves the more intuitive physical unit meter. The altimetry derived from near infrared imaging can be compared to the Magellan GTDR to test whether an orientation model with a 
76

certain period of rotation aligns the two data sets.

\section{Observations}

\subsection{Venus Express VIRTIS}

During the acquisition of the VIRTIS data set the spacecraft Venus Express was in an elliptical orbit around Venus with the apoapsis roughly $60000 \mathrm{~km}$ above the southern pole and periapsis roughly at 100 to $200 \mathrm{~km}$ altitude (Svedhem et al., 2007). VIRTIS is a line scanning spectrometer, the image of a slit is dispersed across a rectangular array of detectors to create a line of adjacent spectra in the range between 0.2 and $5 \mu \mathrm{m}$. A scanning mirror allows repeated acquisition of spectra with varying angles perpendicular to the slit to ultimately construct a three dimensional image cube with two spatial and one spectral dimension (Coradini et al., 1998). The field of view of VIRTIS corresponds to roughly one third of the Venus disc at apoapsis (Drossart et al., 2007) and extensive imaging of the surface is restricted to the southern hemisphere. Two types of observations are used for this study: mosaics of the disc of the planet from apoapsis, and images from the ascending or descending branch of the orbit with spacecraft altitudes greater than $10000 \mathrm{~km}$ (Titov et al., 2006).

The thermal emission from the surface at $1.02 \mu \mathrm{m}$ wavelength is measured on the dark side of the planet. To reduce the impact of stray light from the bright side of Venus, the slit of the instrument is generally oriented parallel to the terminator for the apoapsis mosaics, which results in a correlation of VIRTIS image alignment with referenced longitude, i.e. longitude on average increases from the left side of the images to the right.

The observed angle of emission varies but its influence on radiance is virtually independent from any property of the surface. The surface thermal emission radiation is intensely scattered at air molecules and cloud particles and as a result the anisotropy of the radiation field at the top of the atmosphere is dominated by the upper cloud structure (Grinspoon et al., 1993). This has additional implications for coordinate referencing, as the image of the surface thermal emission appears projected on the cloud layer between 50 and $74 \mathrm{~km}$ altitude (Ignatiev et al., 2009). To account for this, the VIRTIS data set contains two sets of coordinates: one at the intersection of the line of sight with the 
surface reference sphere at the mean planetary radius and one at a reference sphere 60 $\mathrm{km}$ higher, representing the cloud layer. VIRTIS data coordinates are referenced in accordance with the orientation model recommended by the IAU (Seidelmann et al., 2002) which includes a period of rotation of 243.0185 d estimated from Magellan radar images (Davies et al., 1992).

The VIRTIS data processing for surface imaging is described in more detail in the work of Mueller et al. (2008). It includes corrections for stray sunlight, viewing geometry and cloud opacity retrieved from VIRTIS band 30 at approximately $1.31 \mu \mathrm{m}$ wavelength. The notable difference is that here the polynomial fit to the average relation of thermal emission brightness temperature to Magellan topography is not used to predict local radiance from topography, but instead to estimate local topography from VIRTIS radiance.

This estimate of surface topography from top of atmosphere thermal emission radiance is somewhat facilitated by the highly reflective atmosphere, which reduces the influence of emissivity on the radiation measured on the dark side of the planet (Moroz, 2002). The hemispherically integrated reflectance $R$ of the cloud layer is modeled to be on average 0.82 (Hashimoto and Imamura, 2001). Thermal emission radiation is the product of black body radiation at surface temperature $B(T)$ and emissivity $\varepsilon$. The radiation originating from the surface is reflected between atmosphere with reflectivity $R$ and surface with albedo $a=1-\varepsilon$ and the outbound hemispherically integrated radiation flux at the top of the atmosphere $F_{\text {toa }}$ can be approximated by

$$
F_{\text {toa }}=\frac{1-R}{1-R(1-\varepsilon)} \varepsilon \pi B(T)
$$

(Hashimoto and Sugita, 2003). From this equation follows that a deviation of $10 \%$ from an emissivity of 0.85 -typical for basalt- results only in a variation in outbound radiation of 2 to $3 \%$ (Hashimoto and Sugita, 2003). If the surface of Venus typically has a lower emissivity of about 0.6 owing to chemical weathering as proposed by Smrekar et al. (2010), this effect is less pronounced. $10 \%$ emissivity variation then corresponds to about $5 \%$ radiance variation.

A modification of Eq. 1 is used to correct for the variable cloud opacity, yielding for each spectrum a brightness temperature which differs from the surface temperature because of the unknown surface emissivity and extinction in the lowest part of the atmosphere. This brightness temperature monotonically decreases with surface topography. 
A second degree polynomial of all brightness temperature measurements is fitted to the corresponding GTDR altimetry values. This polynomial then allows to estimate the topography for each VIRTIS data point.

The connection between VIRTIS data and Magellan altimetry data is made according to the orientation model recommended by the IAU. In the following other orientation models are evaluated, but the fit is not repeated for each model. A small horizontal misalignment between the two data sets most likely only increases the scatter of Magellan data with respect to the fit but introduces no significant bias.

The data points are extracted from VIRTIS nightside images with an exposure duration of at least $3 \mathrm{sec}$. Data frames -corresponding to one exposure of the slit- with minimum angle between surface normal and direction towards the sun of less than $95^{\circ}$ are excluded from analysis to avoid the sunlight scattered for several degrees beyond the terminator. Spectra with emission angles of more than $85^{\circ}$ have insufficient signal to noise ratio and spatial resolution and are likewise excluded. The images used were acquired between May 2006 and August 2008 with a median date of 9 January 2007.

Fig. 1 a) shows a map representation of the median over time of VIRTIS derived topography data. In general, the data at more equatorial latitudes and in the eastern hemisphere are more sparse due to mission constraints, here the median is less effective in removing noise (Mueller et al., 2008). For the following calculations, the individual VIRTIS measurements are used and not the projected and averaged map representation.

\subsection{Magellan GTDR}

The Magellan GTDR (Version 2.3) used here was reprocessed by Rappaport et al. (1999) to correct for Magellan ephemeris errors. The ephemeris corrections were applied to single orbit altimeter footprints from the Magellan Altimetric and Radiometric Composite Data Record (ARCDR), which is coordinate referenced following the IAU recommendations from 1985 (Davies et al., 1987). The altimeter readings were acquired between August 1990 and August 1992 over three mapping cycles each covering approximately the whole surface though with data gaps. For the creation of the gridded GTDR map the readings were averaged over time and thus the median acquisition time Jan. 8 1991 is taken as representative for all of the GTDR data. 
The Magellan GTDR data has a sampling distance of about $5 \mathrm{~km}$ but the actual spatial resolution varies with latitude between 8 and $27 \mathrm{~km}$ (Ford and Pettengill, 1992). This spatial resolution is however in any case better than the spatial resolution of the VIRTIS data. Near infrared radiation transmitted through the clouds of Venus is diffusely scattered and mixing of radiation from different surface areas reduces the spatial resolution to $\sim 90 \mathrm{~km}$ (Hashimoto and Imamura, 2001). The GTDR data is here used in comparison with VIRTIS data, which requires that the GTDR spatial resolution be reduced to that of VIRTIS. To this end the GTDR is smoothed with a moving weighted average following the algorithm described in Mueller et al. (2008). A projection of this smoothed GTDR data set is presented in Fig. 1 b).

\section{Comparison of the two data sets}

We have visually compared the two maps in Fig. 1 and conclude that the Magellan altimetry appears systematically offset to the west relative to the Venus Express map when following the IAU recommendations. This offset is present at all longitudes and becomes much less obvious towards the south pole. The offset therefore has the general characteristics of a rotation around the planetary spin axis.

The method of least squares provides a straightforward way to estimate both the offset in longitude and error of the offset from the $\chi^{2}$ statistic described in section 3.1 (e.g. Press et al., 1992). This approach, however, does not easily account for systematic errors, e.g. in the VIRTIS coordinate referencing, or non-random errors that are correlated with location such as those arising from the unknown surface emissivity.

Nevertheless, we first proceed with the least squares method to find the offset and to investigate whether the vertical error of VIRTIS derived altimetry allows for a significant estimate of the offset between the data sets. Then the error of the offset is again estimated by using a 'bootstrap' approach (e.g. Press et al., 1992) and by dividing the VIRTIS data set into subsets and finding the offset for each. The latter two methods are more likely to provide an more realistic estimate of the certainty of the result but systematic errors can also additionally impact the accuracy of the result.

The problem of accuracy is approached from another direction by testing the effects of the most probable sources of systematic errors, i.e. surface emissivity variation, VIRTIS 
coordinate referencing and an error in the spin axis direction. We note that these systematic errors not only can impact the accuracy, but also likely increase the uncertainty estimated through the subset and 'bootstrap' methods.

\subsection{Differences between VIRTIS and GTDR}

To estimate the offset, the minimum of the $\chi^{2}$ statistic of the $n \sim 10^{7}$ VIRTIS derived altimetry values $Z_{i}\left(x_{i}\right)$ with respect to the corresponding Magellan altimetry $Z_{\mathrm{MGN}}\left(x_{i}^{\prime}\right)$ is found:

$$
\chi^{2}=\sum_{i=1}^{n}\left[\frac{Z_{i}\left(x_{i}\right)-Z_{\mathrm{MGN}}\left(x_{i}^{\prime}\right)}{\sigma_{\mathrm{VEX}}}\right]^{2}
$$

where $x_{i}$ are the coordinates of VIRTIS data, $x_{i}^{\prime}$ are coordinate transformation of $x_{i}$ including the variable offset, and $\sigma_{\text {VEX }}$ is the error of VIRTIS derived altimetry. The error is expected to vary with pixel position on the detector, instrumental temperature, acquisition exposure duration, observation geometry, cloud opacity and space weather. For convenience we adopt a constant value for all data points.

The minimum is found by calculating $\chi^{2}$ for various offsets corresponding to rotations of the planetary surface. For models with one parameter, the limits of confidence around this minimum are equivalent to an increase in $\chi^{2}$ by one (Press et al., 1992), provided that the VIRTIS deviates from the Magellan data with a normal distribution with variance $\sigma_{\mathrm{VEX}}{ }^{2}$ and has no error in $x_{i}$. When following the IAU recommendations, i.e. assuming no offset $x_{i}=x_{i}^{\prime}$, and further assuming $\chi^{2}=n-1$, Eq. 2 leads to $\sigma_{\mathrm{VEX}} \simeq 2500 \mathrm{~m}$, which can be adopted as data error for the calculation of limits of confidence (Press et al., 1992).

Fig. 2 shows a histogram of the differences between VIRTIS and GTDR altimetry with $x_{i}=x_{i}^{\prime}$. The median of the distribution is at $\sim 60 \mathrm{~m}$ and the 16 th and 84 th percentile are found 500 and $530 \mathrm{~m}$ difference from this value. This is not consistent with the above assumption of a normal distribution with a standard deviation of 2500 $\mathrm{m}$. An overlay of a fitted gaussian with center at $58 \mathrm{~m}$ and standard deviation of 494 $\mathrm{m}$ shows that outlying differences are systematically more frequent than expected in a normal distribution that describes the central 95 percentiles well. This may be due to a non-gaussian distribution or a varying error $\sigma_{\mathrm{VEX}}$. The formal limits of confidence derived from the assumption of normally distributed error described by a constant $\sigma_{\mathrm{VEX}}$ 
might therefore be spurious, which is exacerbated by the possibility of errors in the coordinate referencing of VIRTIS.

Aside from the possible errors in the referencing, a local bias in the derived altimetry can also influence the $\chi^{2}$ statistic. If the bias is correlated with the slope, i.e. the partial derivative of the topography with the coordinate shift, it may appear similar to the bias introduced by an horizontal offset between the two data sets and thus may introduce a bias in the position of the minimum $\chi^{2}$. The map in Fig. 1 a) represents the median over time and therefore gives evidence of any local biases. Various systematic differences are obvious between the map representations of the data sets, which can not be explained by random errors or offsets in coordinates. Some of these correspond to a bias in derived topography of up to $600 \mathrm{~m}$ and have been interpreted to be caused by surface emissivity variation (Helbert et al., 2008; Mueller et al., 2008; Smrekar et al., 2010).

This leads to the question, whether such surface emissivity variations are more influential than the biases introduced by coordinate offsets. For a qualitative evaluation of this problem, two subsets of the VIRTIS data set are selected by the criterion, that the data are acquired at locations where the median over time deviates from the Magellan topography for more than $300 \mathrm{~m}$. The frequency distribution of deviations from the GTDR are also plotted in Fig. 2 a), where the red graphs corresponds to the locations with a bias at least $300 \mathrm{~m}$ lower than Magellan and the blue graphs correspond to a bias of at least $300 \mathrm{~m}$ above the GTDR. The data within subsets exceed the criterion due to random noise plus any combination of a bias in VIRTIS altimetry and horizontal offset to the GTDR.

In Fig. 2 b) the relative frequency distributions of the partial derivatives of topography with respect to longitude are plotted for the whole data set and the two subsets. The subset with a bias towards too low values is offset towards higher frequencies at positive topography derivatives -i.e. western slopes- while the subset with a bias to higher values is offset towards eastern slopes, when compared to the total data set. If assuming an offset of $-0.3 \mathrm{deg}$ in longitude and then reselecting the subsets, the offsets are reduced (Fig. 2). This is consistent with the effect of a coordinate offset in longitude between 0 and $-0.3^{\circ}$.

This may also be due to a correlation of both high emissivity with western slopes 
and low emissivity with eastern slopes, however it seems unlikely that any coincidental emissivity correlation with slope would produce such a symmetrical effect both in high and low emissivity values. The subset with bias to too high values is now more frequent on the steep western slopes, which indicates that the offset of -0.3 deg may be to extreme.

To find the best offset, the minimum of $\chi^{2}$ is found with respect to the transformation $x \rightarrow x^{\prime}$ that aligns the two data sets.

\subsection{Aligning VIRTIS and GTDR}

To connect the two data sets separated in time by 16 years, the coordinates of VIRTIS data are traced back through time using the to be tested set of rotational parameters prescribing the orientation of Venus. The transformation is

$$
x^{\prime}=M^{\top} A R A^{\top} V x
$$

where $x^{\prime}$ and $x$ are VIRTIS data barycentric cartesian coordinates in the frame of GTDR and VIRTIS coordinate referencing. $V$ and $M$ are the transformation matrices from Venus coordinates to Earth mean equatorial coordinates at the epoch of J2000 according to coordinate systems used by VIRTIS (Seidelmann et al., 2002) and the GTDR (Davies et al., 1987), respectively. $A$ is constructed in the same way as $V$ and $M$ but represents the set of rotational parameters to be evaluated. $V$ and $A$ are calculated for the Julian day of VIRTIS observations and $M$ for 8 January 1991, the median data acquisition time of the GTDR. $R$ is a rotation around the pole axis with an angle determined by the number of Julian days between the VIRTIS data acquisition time and 8 January 1991 and the angular velocity of the orientation model under evaluation. The smoothed GTDR topography data corresponding to the VIRTIS data at $x$ are then found through cubic spline interpolation of 16 GTDR points neighboring $x^{\prime}$. To ensure that all estimates are based on the same subset of VIRTIS and GTDR data, only those VIRTIS data are used, which are not within $100 \mathrm{~km}$ distance of missing GTDR data for all the orientation models directly compared with each other.

\subsection{Offset in Longitude}

The first test aims to estimate the offset in longitude between the GTDR and VIRTIS data with the orientation model currently recommended by the IAU (Davies et al., 
1992; Seidelmann et al., 2002) and used for VIRTIS coordinate referencing. Offsets in longitude ranging from $-0.3^{\circ}$ to $0.08^{\circ}$ are added to the VIRTIS data coordinates. The minimum of $\chi^{2}$ is located at a longitude offset of $-0.165^{\circ}$. Visual comparison of the map representations of the data sets confirms that this offsets appears to align VIRTIS and GTDR data.

The sum of the squares of all altimetry deviations between GTDR and VIRTIS at the minimum of $\chi^{2}$ is $\sim 1.1 \cdot 10^{14} \mathrm{~m}^{2}$, with $n=17381826$ this corresponds to a root mean square deviation (RMSD) of $2506 \mathrm{~m}$. If no independent error estimate is available, the minimum of $\sigma_{\mathrm{VEX}}=\sqrt{\chi^{2} /(n-1)}$-approximately equal to the RMSD- can be used to normalize $\chi^{2}$ for an estimate of the limits of confidence of the fit (Press et al., 1992). The error estimated from the RMSD appears exaggerated in comparison with the central 95 percentiles of the deviations (Fig. 2). Adopting the value of $2506 \mathrm{~m}$ as error, $\chi^{2}$ increases by one at a distance of $0.005^{\circ}$ longitude from the minimum. This is a measure of the $1 \sigma$ confidence interval, which however is only then valid if there are only vertical and normally distributed errors in the VIRTIS data.

The large difference between the deviations in the central 95 percentiles (Fig. 2) and the RMSD hints towards the existence of extreme outliers. In order to estimate the robustness of the longitude offset estimate, additional data processing steps are introduced to reduce extreme errors. VIRTIS data calibration by default searches for single pixel spikes and saturated pixels and these are not included in this analysis. In addition to this, VIRTIS derived altimetry deviating more than $7500 \mathrm{~m}$ from the GTDR is not considered for the new data processing. Instrumental stray light and changes in the instrumental spectral transfer function from thermal stresses can introduce a bias that is approximately constant for each VIRTIS image. This bias is approximated by the average of the difference between VIRTIS and GTDR and subtracted from the measurements. The bias is typically around $300 \mathrm{~m}$, but exceeds $2500 \mathrm{~m}$ in two images. No adjustments are made to the coordinates and therefore the subtraction of these biases will partly remove differences introduced by any deviation in the coordinates. The resulting $\chi^{2}$ is therefore biased towards confirming the IAU coordinate referencing recommendations. The resulting minimum $\chi^{2}$ is found at $-0.1541 \pm 0.0010^{\circ}$ longitude relative to the IAU recommendations. The smaller formal confidence interval follows from the smaller RMSD 


\begin{tabular}{cc} 
Orbits & Offset $/{ }^{\circ}$ \\
\hline $25-93$ & -0.143 \\
$94-141$ & -0.170 \\
$141-316$ & -0.269 \\
$317-334$ & -0.113 \\
$334-358$ & -0.305 \\
$359-370$ & -0.127 \\
$370-388$ & -0.091 \\
$388-588$ & -0.150 \\
$588-603$ & -0.189 \\
\hline
\end{tabular}

Table 1: Longitude offsets derived from each of nine subsets. VIRTIS images are assigned to subsets according to data acquisition time so that each subset contains nearly the same amount of data. Venus Express orbital period is $24 \mathrm{~h}$. Orbit insertion was on 4 April 2006

of $569 \mathrm{~m}$ achieved with the additional data processing steps.

For the formal confidence interval it is assumed that the error of every data point is independent. For VIRTIS referencing errors, the error is not independent for all data points in the same image. In this case, an adaption of the "bootstrap" Monte Carlo simulation of the confidence interval described by Press et al. (1992) may give a better estimate. Sample sets of images are drawn randomly with replacement to create a number of sample data sets with the same number of images as the whole data set. Each sample set therefore omits some images and contains images twice or more often. The standard deviation of the position of the minimum $\chi^{2}$ over roughly 1000 of these sample sets is $0.01^{\circ}$, a magnitude larger than the formal confidence interval of the $\chi^{2}$ statistic but still an order of magnitude smaller than the observed offset.

Dividing the data set into similarly sized subsets based on time of data acquisition may provide insight into the certainty of the observed offset and additionally allows to determine if the offset varies significantly with time. The resulting fitted offsets for nine subsets are plotted in Fig. 3 with confidence intervals derived through the 'bootstrap' method. The offsets are not consistent with each other but can not be very plausibly attributed to a real movement of the planet. A more likely explanation for the variance 
of the fitted offset are systematic errors. For the certainty of the observed offset the standard deviation of the offset in the nine subsets of $0.071^{\circ}$ is adopted. This confidence interval corresponds to an increase of the $\chi^{2}$ statistic by 2836 .

This high $\chi^{2}$ increase over the confidence interval estimated from the subset method indicates that the vertical random error of the VIRTIS derived altimetry only plays a very minor role for the uncertainty of the offset. This means the $\chi^{2}$ statistic is not meaningful for the significance of our result. In the following we will evaluate models on their RMSD, which may be more intuitive. The error of $0.071^{\circ}$ longitude derived from the subset method corresponds to an increase of the RMSD ( $\triangle \mathrm{RMSD})$ of $0.046 \mathrm{~m}$.

While the offset is supported by all of the the subsets, the question remains whether any systematic error affects all of the data to consistently produce a similar offset. In the following several possible systematic errors are investigated.

\subsection{Influence of surface emissivity}

Surface thermal emission anomalies thought to be unweathered lava flows at the flanks of volcanic structures (Helbert et al., 2008; Mueller et al., 2008; Smrekar et al., 2010) introduce a bias of up to $600 \mathrm{~m}$ in the derived altimetry. The three strongest anomalies are at Juturna and Cavilaca fluctūs on the southern flank of the Lada Terra rise (Helbert et al., 2008), at the summit and northeastern flank of Idunn mons in Imdr regio and at the western flank of Shiwanokia corona (Smrekar et al., 2010).

As these anomalies are on the flanks of topographic features the position of the topographic feature may appear offset in the near infrared altimetry. The anomalies are found in various directions relative to the topographic features but overall the $\chi^{2}$ statistic might be biased if the distribution of emissivity anomalies with respect to slope direction is by coincidence not symmetrical (Fig. 2 b and c).

To better understand the possible influence of surface emissivity variation, data within four areas containing the strongest anomalies with excess thermal emission are removed from the data set. The areas are Imdr regio (bounded by $50^{\circ} \mathrm{S}, 40^{\circ} \mathrm{S}, 210^{\circ} \mathrm{E}$ and $220^{\circ} \mathrm{E}$ ), Themis regio $\left(50^{\circ} \mathrm{S}, 30^{\circ} \mathrm{S}, 270^{\circ} \mathrm{E}, 300^{\circ} \mathrm{W}\right)$, Dione regio $\left(40^{\circ} \mathrm{S}, 30^{\circ} \mathrm{S}, 320^{\circ} \mathrm{E}, 330^{\circ} \mathrm{E}\right)$ and Lada Terra together with the south-eastern rim of the Lavinia basin $\left(80^{\circ} \mathrm{S}, 40^{\circ} \mathrm{S}, 340^{\circ} \mathrm{E}\right.$, $\left.20^{\circ} \mathrm{E}\right)$. These areas encompass all of the volcanic hotspot centers identified in Magellan 
gravity data of the southern hemisphere (Smrekar, 1994; Stofan et al., 1995), and are thus areas with a high likelihood of ongoing active volcanism.

The data set excluding these areas has approximately $20 \%$ less data points. The minimum of the $\chi^{2}$ statistic is at $-0.1291^{\circ}$ longitude relative to IAU recommendations with a formal confidence interval of $0.0012^{\circ}$. The $20 \%$ wider confidence interval compared to the full data set may be due to fewer data and much less topographic features (see Fig. 1). The standard deviation of the fitted longitude offset in nine subsets is $0.066^{\circ}$. Compared to the $0.071^{\circ}$ of the full data set, this indicates that the systematic errors are not efficiently removed with the exclusion of the four areas.

We are not aware of any effects possibly causing a systematic bias of surface thermal emission on the eastern or western slope of topographic highs. Orographic effects of surface temperature or weathering might play a role but aeolian features indicate that the prevailing surface winds are in North South direction (Greeley et al., 1995).

The Magellan radiothermal emission measurements at $12.9 \mathrm{~cm}$ wavelength have revealed anomalous emissivity at high altitudes above 4 to $5 \mathrm{~km}$ (Pettengill et al., 1992). This anomaly is thought to be caused by a highly dielectric mineral that is only stable below a certain temperature, possibly influenced by atmospheric composition (e.g. Fegley et al., 1997; Wood, 1997). The altitude of this 'snowline' varies with latitude but no bias with direction of topographic slope is reported, even when the 'snowline' was used as control on stereo image digital elevation models (Arvidson et al., 1994; Howington-Kraus et al., 2002). These radiothermal emissivity anomalies are however not relevant for the VIRTIS derived infrared emissivity data. VIRTIS coverage is restricted to parts of the southern hemisphere with negligible surface area above $4 \mathrm{~km}$ altitude.

In conclusion, the most strongest thermal emission anomalies influence the fit of the offset only by $0.025^{\circ}$. Less obvious thermal emission anomalies can further influence the fit, however it is unlikely that such more subtle anomalies could influence the fit more by coincidence. The existence of a systematic emissivity difference between eastern and western flanks of topographic highs appears unlikely.

\subsection{VIRTIS coordinate referencing}

A simple explanation for the observed offset in longitude would be a systematic error in the coordinate referencing of the data sets such as from misalignment of the instrument 
or refraction in the atmosphere. The typical viewing geometry of VIRTIS nightside observations from above the south pole with the slit oriented parallel to the terminator means that the planetary coordinates are correlated with the instrument and spacecraft reference frame. The average difference of referenced longitude between neighboring pixels is $0.24^{\circ} /$ pixel in the direction of the slit (i.e. the spacecraft $y$-axis as defined in (Titov et al., 2006)) and $0.01^{\circ} /$ pixel perpendicular to the slit (i.e. the spacecraft x-axis as defined in (Titov et al., 2006)). Star and limb observations with VIRTIS exclude a misalignment greater than 0.4 pixel in the slit direction and 1.3 pixel perpendicular to the slit, corresponding to an error in longitude referencing of $0.1^{\circ}$ in the worst case.

A misalignment of 0.1 pixel, i.e. $0.25 \mathrm{mrad}$, in either direction is simulated by interpolating between the referenced coordinates of neighboring pixels. The fitted offset increases with misalignment of the instrument along the y-axis of the spacecraft, and decreases with misalignment along the x-axis. The modeled misalignment of 0.1 pixel along the $y$-axis results in a fitted longitude offset of $0.0164^{\circ}$, and along the $\mathrm{x}$-axis in an offset of $0.0007^{\circ}$.

This offset from modeled misalignment is smaller than expected from the average differences of longitude between neighboring pixels. This might be due to the weighting introduced by the distribution of topographical features. Topographical features are scarce at lower latitudes where the effect of a misalignment for longitude referencing is greater. The maximally possible longitude bias of $4 \cdot 0.0164^{\circ}+13 \cdot 0.0007^{\circ}=0.075^{\circ}$ from a biased instrument misalignment is similar to the observed variation of the longitude offset over nine data subsets with a standard deviation of $0.071^{\circ}$. The possible misalignment may therefore have a significant effect on the observed offset, although it can not explain the full offset of $0.154^{\circ}$.

To account for the light scattering atmosphere, VIRTIS data is referenced to a sphere with radius $6112 \mathrm{~km}$ representing the cloud layer of Venus, equivalent to $60 \mathrm{~km}$ altitude above the mean planetary radius. The altitude of optical depth of one is $74 \pm 1 \mathrm{~km}$ at low latitudes on the dayside, and decreases below $-50^{\circ}$ latitude to a variable altitude with an observed minimum of $63 \mathrm{~km}$ at the south pole (Ignatiev et al., 2009). The cloud base was found by nephelometer and particle counter experiments on descent probes and is expected between 45 and $50 \mathrm{~km}$ altitude (Ragent et al., 1985). The reference altitude 
therefore lies roughly in the middle of the clouds. Nevertheless, a different altitude may lead to a more appropriate referencing of the surface image projected on the clouds.

The difference $h$ of this best reference sphere to the altitude of $60 \mathrm{~km}$ then causes a local distortion in the coordinate referencing of VIRTIS data. The referenced and the most appropriate coordinates are both on a line perpendicular to the limb and the angle $\alpha$ between them as seen from the instrument is approximately

$$
\alpha=\frac{h}{s} \sin \theta
$$

where $s$ is the slant distance between spacecraft and reference sphere and $\theta$ is the emission angle. This allows us to calculate the bias in longitude referencing introduced by an inappropriate cloud altitude by linear interpolation of the longitudes of VIRTIS geometry data to the lines of sight with correct coordinates. Assuming a cloud altitude error $h$ of $14 \mathrm{~km}$, the average bias in longitude referencing is $-0.004^{\circ}$. Assuming $h=-10$ $\mathrm{km}$ the average deviation is $0.004^{\circ}$, with minimum value of $-0.49^{\circ}$, maximum value of $0.46^{\circ}$ and a standard deviation of $0.04^{\circ}$. The bias in latitude corresponding to $h$ of $14 \mathrm{~km}$ is $0.09^{\circ}$. This small dependence of longitude referencing on the reference sphere radius is due to the typical viewing geometry from above the south pole.

This estimate of coordinate bias is verified by referencing the data to a spheres with $6102 \mathrm{~km}$ and $6122 \mathrm{~km}$ radius, corresponding to the lower cloud and upper cloud at 50 $\mathrm{km}$ and $70 \mathrm{~km}$ altitude. The resulting fitted offsets are $-0.157^{\circ}$ and $-0.152^{\circ}$, respectively, both within $0.003^{\circ}$ of the longitude offset of $-0.154^{\circ}$ at $60 \mathrm{~km}$ altitude. The altitude of the reference sphere within the cloud layer does therefore not significantly affect the fit of the longitude offset.

\subsection{Rotation axis direction}

A deviation in the parameters describing the direction of the rotation axis can appear similar to an offset in longitude. To investigate this effect for the VIRTIS data set, the rotation axis parameters derived by Davies et al. (1992) and recommended by the IAU (Seidelmann et al., 2002) are varied by $2 \sigma$ and the best fitting longitude offset aligning the VIRTIS and Magellan altimetry data sets is found by minimizing the RMSD. The results are presented in Tab. 2. The fitted longitude offset is sensitive to right ascension, 


\begin{tabular}{l|l|l|l} 
& \multicolumn{3}{|l}{ Fit at right ascension: } \\
Declination & $272.72^{\circ}$ & $272.76^{\circ}$ & $272.80^{\circ}$ \\
\hline $67.14^{\circ}$ & $-0.168^{\circ}$ & $-0.156^{\circ}$ & $-0.145^{\circ}$ \\
& $0.183 \mathrm{~m}$ & $0.189 \mathrm{~m}$ & $0.212 \mathrm{~m}$ \\
$67.16^{\circ}$ & $-0.166^{\circ}$ & $-0.154^{\circ}$ & $-0.142^{\circ}$ \\
& $-0.003 \mathrm{~m}$ & $0.000 \mathrm{~m}$ & $0.020 \mathrm{~m}$ \\
$67.18^{\circ}$ & $-0.164^{\circ}$ & $-0.152^{\circ}$ & $-0.140^{\circ}$ \\
& $-0.132 \mathrm{~m}$ & $-0.132 \mathrm{~m}$ & $-0.114 \mathrm{~m}$ \\
\hline
\end{tabular}

Table 2: Influence of variation of rotation axis on fitted offset in longitude and the corresponding change of the minimum root mean square deviation between the data sets, relative to the value of $569.263 \mathrm{~m}$ for the IAU orientation model with longitude offset. The rotation axis right ascension (RA) and declination $(\mathrm{DE})$ is varied around the values recommended by the $\mathrm{IAU}\left(\mathrm{RA}=272.76^{\circ}, \mathrm{DE}=67.16^{\circ}\right.$ ) for $2 \sigma$ of the stated error in the work of Davies et al. (1992).

however a deviation of more than $2 \sigma$ from the values recommended by the IAU is required to explain the offset in longitude. Assuming that the trend is linear, a deviation of about $20 \sigma$ could explain the offset in longitude.

The minimum $\chi^{2}$ hint towards a higher spin axis declination than recommended by the IAU. It is desirable to find the minimum RMSD in the parameter space of pole right ascension, declination and longitude offset. However, the possibly large systematic errors in VIRTIS latitude referencing and the uneven distribution of data over the planet reduce confidence in the accuracy of the fit for all parameters. We did not attempt this fit due to the large computational cost compared to the doubtful outcome.

The fit of the longitude offset is not significantly affected by plausible errors in the position of the pole recommended by the IAU. This spin axis direction is confirmed by independent estimates based on Earth based radar observations and Magellan gravity observations, which however led to significantly different periods of rotation (Davies et al., 1992; Konopliv et al., 1999).

\subsection{Rotation period}

If this offset in longitude is due to a deviation in rotation period, the sign indicates a slower retrograde rotation than recommended by the IAU. The difference of median data 
acquisition times is 5845 Julian days. The difference of angular velocity corresponding to an offset in longitude of $-0.154 \pm 0.071^{\circ}$ is $-2.6 \cdot 10^{-5} \pm 1.2 \cdot 10^{-5} \circ /$ day. The angular velocity of the orientation model is $1.4813688^{\circ}$ /day, adding the offset leads to a period of rotation of $243.0228 \pm 0.0020$ days.

We can not confirm or reject this period of rotation from observation of the evolution of the longitude offset. If the true period of rotation of Venus is 243.023 days, the offset in longitude occurring over the 600 days of VIRTIS observations is approximately $0.02^{\circ}$, which is small compared to the scatter of the longitude offsets of the 9 subsets of $0.071^{\circ}$. The offsets of the subsets do not appear to have a significant trend (Fig. 3).

The RMSD of the orientation model with a revised period of rotation of 243.023 days is $0.001 \mathrm{~m}$ higher than the orientation model with a constant offset of $-0.154^{\circ}$ longitude (see table 3). This indicates a worse fit, which is however not significant compared to the $\triangle$ RMSD adopted as limit of confidence. In other words, the observed offset is consistent with a revised period of rotation of 243.0228 days, but we can not show that the offset changes accordingly over the 2 years of VIRTIS observations. There is however a reason why the revised period of rotation is more plausible than the constant offset as detailed in the next section.

\subsection{Other sets of rotational parameters}

The period of rotation of $243.0228 \pm 0.0020 \mathrm{~d}$ is consistent with the estimates from ground based observations by Slade et al. (1990), Shapiro et al. (1990) and reported by Davies et al. (1992), as well as to the value derived from comparison of Venera 15/16 and Magellan radar images by Davies et al. (1992). It is not consistent with the estimates based on Magellan SAR data alone (Davies et al., 1992) or Magellan gravity data Konopliv et al. (1999).

The $\chi^{2}$ values for several models are listed in table 3 in order of increasing $\chi^{2}$. Appended to the table are two hybrid models based the previous tests, derived from the orientation model recommended by the IAU (Davies et al., 1992; Seidelmann et al., 2002). The first hybrid model adds a constant longitude offset of $-0.154^{\circ}$ and the second uses a revised period of rotation of $243.023 \mathrm{~d}$, matched to introduce an similar offset at the median VIRTIS data acquisition time. 
The model with the constant offset provides the reference RMSD of $569.262 \mathrm{~m}$ at the offset of $-0.154^{\circ}$ longitude. A measure of the significance of $\Delta$ RMSD can be derived from the deviation of the fitted shifts of nine subsets of $0.071^{\circ}$, which corresponds to an increase in RMSD of 0.046. This confidence limit estimate however assumes that the spin axis is well known. If the spin axis direction is varied by $2 \sigma$ in both right ascension and declination the minimum RMSD changes by as much as $0.212 \mathrm{~m}$.

The best fit is achieved with the set derived from Goldstone ground based radar observations (Slade et al., 1990). Comparison with table 2 indicates that much of the $\triangle$ RMSD can be attributed spin axis declination, which deviates significantly from other estimates. The second best fit comes from the orientation model based on all available Earth based data from 1972 to 1988 credited to G.H. Pettengill in the work of Davies et al. (1992). This model is within its stated error consistent with IAU recommendations for the spin axis (Seidelmann et al., 2002) and a revised rotation period of 243.023 days, as derived from the fitted offset in longitude between VIRTIS and Magellan data. The spin axis is furthermore consistent with the Magellan gravity model (Konopliv et al., 1999).

Although the VIRTIS Magellan comparison RMSD may suggest otherwise, the model based on the Earth based 1972 to 1988 observations is probably preferable to the Goldstone 1972 to 1982 model. As stated above, the VIRTIS referencing may contain systematic errors in latitude that have the potential to affect the fit when varying the position of the pole.

\section{Discussion and Conclusions}

Over the 16 years between the Magellan and Venus Express missions, an offset in longitude of $0.154 \pm 0.071^{\circ}$ between the two topography data sets is observed when the orientation model following IAU recommendations (Davies et al., 1992; Seidelmann et al., 2002 ) is used. This deviation is relevant for the retrieval of surface emissivity from orbiter near-infrared imaging (Helbert et al., 2008; Hashimoto et al., 2008; Arnold et al., 2008; Mueller et al., 2008; Smrekar et al., 2010; Haus and Arnold, 2010).

The orientation model recommended by the IAU (Davies et al., 1992) is based on Magellan synthetic aperture radar (SAR) images and there could conceivably be an offset 


\begin{tabular}{|c|c|c|c|c|}
\hline Observations and Reference & $\begin{array}{l}\text { Period of } \\
\text { rotation } \\
\text { /days }\end{array}$ & $\begin{array}{l}\text { Right } \\
\text { ascension } \\
\text { /deg }\end{array}$ & $\begin{array}{l}\text { Declination } \\
/ \operatorname{deg}\end{array}$ & $\Delta \mathrm{RMSD} / \mathrm{m}$ \\
\hline $\begin{array}{l}\text { Goldstone } 1972 \text { to } 1982 \\
\text { (Slade et al., 1990) }\end{array}$ & $243.022(3)$ & $272.79(14)$ & $67.23(5)$ & -0.198 \\
\hline $\begin{array}{l}\text { Earth based } 1972 \text { to } 1988 \\
\text { (Davies et al., 1992) }\end{array}$ & $243.022(2)$ & $272.74(2)$ & $67.17(2)$ & -0.060 \\
\hline $\begin{array}{l}\text { Earth based }{ }^{(1)} \text { (Davies } \\
\text { et al., 1987, 1992) }\end{array}$ & $243.025(2)$ & $272.69(9)$ & $67.17(6)$ & -0.007 \\
\hline $\begin{array}{l}\text { Magellan gravimetry } \\
\text { (Konopliv et al., 1999) }\end{array}$ & $243.0200(2)$ & $272.743(2)$ & $67.156(1)$ & 0.199 \\
\hline $\begin{array}{l}\text { Magellan SAR }{ }^{(2)} \text { (Davies } \\
\text { et al., 1992) }\end{array}$ & $243.0185(1)$ & $272.76(2)$ & $67.16(1)$ & 0.364 \\
\hline $\begin{array}{l}\text { Venera \& Magellan SAR } \\
\text { (Davies et al., 1992) }\end{array}$ & $243.023(1)$ & $272.43(5)$ & $67.16(2)$ & 0.599 \\
\hline $\begin{array}{l}\text { Earth based } 1975 \text { to } 1983 \\
\text { (Shapiro et al., 1990) }\end{array}$ & $243.026(6)$ & $272.73(9)$ & $67.11(9)$ & 0.681 \\
\hline$-0.154^{\circ}$ longitude offset & 243.0185 & 272.76 & 67.16 & 0 \\
\hline Revised period of rotation & 243.0230 & 272.76 & 67.16 & 0.001 \\
\hline
\end{tabular}

Table 3: Sets of Venus rotational parameters in the epoch of J2000 and their difference in root mean square deviation $\triangle \mathrm{RMSD}$, relative to $\mathrm{RMSD}=568.262 \mathrm{~m}$. The numbers in brackets give error estimates for the last digit or digits. (1) Values recommended by the IAU (Davies et al., 1987) and used for Magellan altimetry referencing (Rappaport et al., 1999). (2) Values recommended by the IAU (Seidelmann et al., 2002) and used for VIRTIS referencing. 
in referencing of the Magellan altimetry relative to the images. Howington-Kraus et al. (2002) test the Magellan sensor model including corrections for refraction and ephemeris errors by fitting radar image stereo pairs and minimizing the residuals between the stereo elevation models and Magellan GTDR altimetry. They report an error in the refraction correction corresponding to $0.15 \mathrm{~km}$ on ground - equivalent to less than $0.01^{\circ}$ longitude at latitudes lower than $80^{\circ}$ - but no systematic deviation between Magellan altimetry and radar imagery.

The offset in longitude could also be due to systematic or random errors in the VIRTIS data set. Excluding some areas which are thought to contain surface emissivity anomalies at recent lava flows (Smrekar et al., 2010) reduces the fitted offset by $0.025^{\circ}$ to $-0.129^{\circ}$. The coordinate referencing error in longitude from instrument alignment may be as large as $0.075^{\circ}$ while the error from uncertainty in the correction for atmospheric refraction is less than $0.003^{\circ}$. The observation of an offset is reproducible with subsets of the VIRTIS data, which additionally allows to an estimate of the error of the offset. After the division into nine subsets the standard deviation of the fitted offsets is $0.071^{\circ}$, which is comparable to the error estimate from VIRTIS referencing. Added together the systematic errors can nearly match the observed offset and if there are yet unidentified systematic errors this might explain the whole offset.

However, the offset can also be introduced if the period of rotation of Venus is 243.0228 days as opposed to the value of 243.0185 days assumed for coordinate referencing based on IAU recommendations. This matches the period of rotation of $243.022 \pm 0.002$ days derived from all available Earth based observations from 1972 to 1988 (Davies et al., 1992) and the period of rotation of $243.022 \pm 0.003$ days derived from Goldstone observations (Slade et al., 1990). The latter orientation model appears to fit VIRTIS and Magellan data better but this is possibly caused by an error in the spin axis direction of the model and a bias in latitude in the VIRTIS data.

The spin axis direction of the former, from the Earth observations with the longest time baseline from 1972 to 1988, agrees with that from Magellan SAR (Davies et al., 1992) and gravity observations (Konopliv et al., 1999). Therefore the three independent spin axis estimates with the smallest formal errors are consistent with each other (Table 3). The rotation periods of these models are however inconsistent or nearly inconsistent 
with each other.

The inconsistency between the estimates of the period of rotation is puzzling, however the estimates are based on data from different times and over different timescales (see Fig. 4). A change in spin rate of this magnitude is not inconsistent with Earth-based radar measurements of the instantaneous spin rate of Venus (Margot et al., 2006) obtained between 2004 and 2009 [Margot, personal communication, 2010].

Therefore it might be possible that the long time baseline estimates represent the average spin rate while the Magellan radar and gravity observations were made during a time when the spin rate deviated from its average. All discussed estimates that do not exclusively use Magellan data have a time baseline of at least 8 years and are formally consistent with a period of rotation of $243.023 \pm 0.002$ days. The Magellan radar (Davies et al., 1992) and gravity (Konopliv et al., 1999) estimates are not consistent with this value, but observe each a 2 year period between 1990 and 1994. Thus a short, singular or periodic length of day excursion could explain why the Magellan radar period of rotation estimate differs by $\sim 5$ min from the estimates with longer time baselines.

A possible explanation for such spin period variations is angular momentum exchange between the solid body of Venus and its thick, superrotating atmosphere (e.g. Schubert, 1983). Assuming relative atmospheric angular momentum exchanges similar to Earth (Hide et al., 1980), length of day variations about one hour are possible (Golitsyn, 1982; Schubert, 1983). Parish et al. (2011) find in a Venus atmosphere general circulation model an angular momentum oscillation with an amplitude of $5 \%$ with a periodicity of $\sim 10$ years. This corresponds to a length of day variation amplitude on the order of $\sim 15$ min (Schubert, 1983).

If the periods of rotation in table 3 are taken as average over each time baseline it is possible to fit the data with corresponding time averages of a sinusoid representing deviations from a period of rotation of $243.022 \mathrm{~d}$. The sinusoid with a period of 10 years and length of day variation amplitude of $15 \mathrm{~min}$ does not result in a good fit for any phase. For sinusoids with a period of 10 years there is a local minimum of the $\chi^{2}$ statistic at an amplitude of 5.4 min and phase of 3.33 radian relative to the year 0 . This minimum $\chi^{2}$ is 3.17 which is consistent with the data errors from table 3 and an appropriate model fitted with five degrees of freedom (Press et al., 1992). 
There are however many formally better fits with sinusoid periods of less than 10 years. Some of these have improbably low $\chi^{2}$ which either hints towards exaggerated error estimates (Press et al., 1992) or towards a problem that is underconstrained due to the data representing averages over time. The deviation of the rotation period when averaged over a time interval greater than the period of the angular momentum oscillation is less than $1 /(2 \pi)$ of the sinusoid amplitude. The errors of the rotation period estimates with baselines greater 8 years are only one order of magnitude smaller than a length of day variation amplitude of $15 \mathrm{~min}$. Thus, for the long baseline estimates, any plausible deviation is therefore very close to or even less than the error. If only the two Magellan estimates with baselines of 2 years contribute significantly to the fit, it is difficult to constrain a sinusoid with three parameters.

While the period and amplitude of length of day variation observed in the global circulation model by Parish et al. (2011) is not consistent with the observations, it is possible that the model does not perfectly represent the atmosphere of Venus and that there is actually a different periodic length of day variation consistent with the observations.

The atmosphere is however not the the only possible source of angular momentum variation. Cottereau et al. (2011) compare various possible contributions to the Venus length of day variation. They conclude that torque exerted by the Sun on Venus represented as a triaxial ellipsoid is the dominating contribution with a length of day variation of $120 \mathrm{~s}$ with a dominant periodicity of $58 \mathrm{~d}$. From global circulation numerical models they derive an atmospheric contribution to the length of day variation of less than a minute with dominant frequencies corresponding to periods of less than 266 days. The numerical models are stated to be similar to the model presented by Lebonnois et al. (2010), which however does not show decadal variations similar to the model of Parish et al. (2011). In total the peak to peak length of day variations modeled by Cottereau et al. (2011) are approximately 3 min and thus additional sources of length of day variation may be required.

Another aspect of the rotation dynamics of Venus is the proximity of rotation period to a resonance with Earth conjunctions at 243.16 days (e.g. Shapiro et al., 1979, 1990). The value of 243.023 days is outside of the interval of rotation periods that can be attained by libration (Shapiro et al., 1990). On the other hand, Caudal (2010) puts forward the 
hypothesis of a differentially rotating solid inner core in resonance with Earth, which again leads to the question of angular momentum exchange.

Investigation of the possible periodicity of the Venus length of day variation is not possible with the data used here. A reinvestigation of the radar feature tracking data with detailed consideration of the times when individual features were observed while allowing for length of day variation may yield better results but is beyond the scope of this manuscript. Additional measurements of the instantaneous spin rate of Venus (Margot et al., 2006) would be very helpful.

Regardless of the large uncertainties of the VIRTIS Magellan comparison, measurements with a shorter time baseline such as the work of Davies et al. (1992) may be less well suited to create a model of planetary rotation for the purpose of coordinate referencing. If we construct a new orientation model using the IAU pole position (Seidelmann et al., 2002) and the Magellan-VIRTIS rotation period obtained here, we find that this model is consistent with the with the model from Earth based observations between 1972 to 1988 as cited in the work of Davies et al. (1992). Both have relatively long time baselines of 16 years and therefore likely provide a more accurate long term description of the orientation of Venus than the model recommended by the IAU (Seidelmann et al., 2002), which is based on radar observations over a period of 2 years (Davies et al., 1992).

\section{Acknowledgements}

Michael Misun and Marita Wählisch made the authors aware of the discrepancy between the coordinate systems of Magellan GTDR and Venus Express VIRTIS. Ellen Stofan and Sue Smrekar noticed the residual bias after nominal alignment of the two coordinate systems. N.T.M. wishes to thank Marita Wählisch, Hauke Hussmann, Frank Scholten, Jürgen Oberst, Paul Chodas and Alexander Konopliv for discussions on the topics of cartographic coordinates and the rotation of Venus. Two anonymous reviewers contributed suggestions that greatly improved this manuscript.

This research has been supported by the Helmholtz Association through the research alliance "Planetary Evolution and Life". We acknowledge the financial support for the VIRTIS instrument from ASI and CNES. We are grateful to the whole VIRTIS on Venus Express team and to Peter G. Ford for providing these excellent data sets. 


\section{References}

Arnold, G., Haus, R., Kappel, D., Drossart, P., Piccioni, G., Oct. 2008. Venus surface data extraction from VIRTIS/Venus Express measurements: Estimation of a quantitative approach. Journal of Geophysical Research (Planets) 113, doi:10.1029/2008JE003087.

Arvidson, R. E., Brackett, R. A., Shepard, M. K., Isenberg, N. R., Fegley, Jr., B., Nov. 1994. Microwave signatures and surface properties of Ovda Regio and surroundings, Venus. Icarus 112, 171-186.

Barsukov, V. L., Basilevsky, A. T., Burba, G. A., Bobinna, N. N., Kryuchkov, V. P., Kuzmin, R. O., Nikolaeva, O. V., Pronin, A. A., Ronca, L. B., Chernaya, I. M., Shashkina, V. P., Garanin, A. V., Krushky, E. R., Markov, M. S., Sukhanov, A. L., Kotelnikov, V. A., Rzhiga, O. N., Petrov, G. M., Alexandrov, Y. N., Sidorenko, A. I., Bogomolov, A. F., Skrypnik, G. I., Bergman, M. Y., Kudrin, L. V., Bokshtein, I. M., Kronrod, M. A., Chochia, P. A., Tyuflin, Y. S., Kadnichansky, S. A., Akim, E. L., Sep. 1986. The geology and geomorphology of the Venus surface as revealed by the radar images obtained by Veneras 15 and 16. Journal of Geophysical Research 91, 378.

Caudal, G. V., Jul. 2010. Hypothesis of a spin-orbit resonance between the Earth and Venus's core. Journal of Geophysical Research (Planets) 115, doi:10.1029/2009JE003370.

Coradini, A., Capaccioni, F., Drossart, P., Semery, A., Arnold, G., Schade, U., Angrilli, F., Barucci, M. A., Bellucci, G., Bianchini, G., Bibring, J. P., Blanco, A., Blecka, M., Bockelee-Morvan, D., Bonsignori, R., Bouye, M., Bussoletti, E., Capria, M. T., Carlson, R., Carsenty, U., Cerroni, P., Colangeli, L., Combes, M., Combi, M., Crovisier, J., Dami, M., DeSanctis, M. C., DiLellis, A. M., Dotto, E., Encrenaz, T., Epifani, E., Erard, S., Espinasse, S., Fave, A., Federico, C., Fink, U., Fonti, S., Formisano, V., Hello, Y., Hirsch, H., Huntzinger, G., Knoll, R., Kouach, D., Ip, W. H., Irwin, P., Kachlicki, J., Langevin, Y., Magni, G., McCord, T., Mennella, V., Michaelis, H., Mondello, G., Mottola, S., Neukum, G., Orofino, V., Orosei, R., Palumbo, P., Peter, G., Pforte, B., Piccioni, G., Reess, J. M., Ress, E., Saggin, B., Schmitt, B., Stefanovitch, Stern, A., Taylor, F., Tiphene, D., Tozzi, G., Oct. 1998. VIRTIS : an imaging spectrometer for the Rosetta mission. Planetary and Space Science 46, 1291-1304.

Cottereau, L., Rambaux, N., Lebonnois, S., Souchay, J., Jul. 2011. The various contributions in Venus rotation rate and LOD. Astronomy and Astrophysics 531, doi:10.1051/0004-6361/201116606.

Davies, M. E., Abalakin, V. K., Bursa, M., Lederle, T., Lieske, J. H., Rapp, R. H., Seidelmann, P. K., Sinclair, A. T., Teifel, V. G., Tjuflin, Y. S., 1987. Report of the IAU/IAG/COSPAR Working Group on Cartographic Coordinates and Rotational Elements of the Planets and Satellites: 1985. Celestial Mechanics 39, 103-113.

Davies, M. E., Colvin, T. R., Rogers, P. G., Chodas, P. W., Sjogren, W. L., Akim, E. L., Stepaniants, V. A., Vlasova, Z. P., Zakharov, A. I., Aug. 1992. The rotation period, direction of the north pole, and geodetic control network of Venus. Journal of Geophysical Research 97, 13141-13151.

Davies, M. F., Abalakin, V. K., Duncombe, R. L., Masursky, H., Morando, B., Owen, T. C., Seidelmann, P. K., Sinclair, A. T., Wilkins, G. A., Cross, C. A., Oct. 1980. Report of the IAU working group on cartographic coordinates and rotational elements of the planets and satellites. Celestial Mechanics 22, 205-230. 
Drossart, P., Piccioni, G., Adriani, A., Angrilli, F., Arnold, G., Baines, K. H., Bellucci, G., Benkhoff, J., Bézard, B., Bibring, J.-P., Blanco, A., Blecka, M. I., Carlson, R. W., Coradini, A., di Lellis, A., Encrenaz, T., Erard, S., Fonti, S., Formisano, V., Fouchet, T., Garcia, R., Haus, R., Helbert, J., Ignatiev, N. I., Irwin, P. G. J., Langevin, Y., Lebonnois, S., Lopez-Valverde, M. A., Luz, D., Marinangeli, L., Orofino, V., Rodin, A. V., Roos-Serote, M. C., Saggin, B., Sanchez-Lavega, A., Stam, D. M., Taylor, F. W., Titov, D., Visconti, G., Zambelli, M., Hueso, R., Tsang, C. C. C., Wilson, C. F., Afanasenko, T. Z., Oct. 2007. Scientific goals for the observation of Venus by VIRTIS on ESA/Venus express mission. Planetary and Space Science 55, 1653-1672.

Dyce, B. R., Pettengill, G. H., Shapiro, I. I., Apr. 1967. Radar determination of the rotations of Venus and Mercury. Astronomical Journal 72, 351-359.

Fegley, Jr., B., Klingelhöfer, G., Lodders, K., Widemann, T., 1997. Geochemistry of Surface-Atmosphere Interactions on Venus. In: Bougher, S. W., Hunten, D. M., Philips, R. J. (Eds.), Venus II: Geology, Geophysics, Atmosphere, and Solar Wind Environment. pp. 591-636.

Ford, P. G., Pettengill, G. H., Aug. 1992. Venus topography and kilometer-scale slopes. Journal of Geophysical Research 97, 13103-13114.

Goldstein, R. M., Feb. 1964. Venus Characteristics by Earth-Based Radar. Astronomical Journal 69, $12-18$.

Goldstein, R. M., Carpenter, R. L., Mar. 1963. Rotation of Venus: Period Estimated from Radar Measurements. Science 139, 910-911.

Golitsyn, G. S., Apr. 1982. The Structure of the Atmospheric Circulation on Venus and Possible Irregularities in its Rotation Rate. Soviet Astronomy Letters 8, 170-172.

Greeley, R., Bender, K., Thomas, P. E., Schubert, G., Limonadi, D., Weitz, C. M., Jun. 1995. Windrelated features and processes on Venus: Summary of Magellan results. Icarus 115, 399-420.

Grinspoon, D. H., Pollack, J. B., Sitton, B. R., Carlson, R. W., Kamp, L. W., Baines, K. H., Encrenaz, T., Taylor, F. W., Jul. 1993. Probing Venus's cloud structure with Galileo NIMS. Planetary and Space Science 41, 515-542.

Hashimoto, G. L., Imamura, T., Dec. 2001. Elucidating the Rate of Volcanism on Venus: Detection of Lava Eruptions Using Near-Infrared Observations. Icarus 154, 239-243.

Hashimoto, G. L., Roos-Serote, M., Sugita, S., Gilmore, M. S., Kamp, L. W., Carlson, R. W., Baines, K. H., Dec. 2008. Felsic highland crust on Venus suggested by Galileo Near-Infrared Mapping Spectrometer data. Journal of Geophysical Research (Planets) 113, doi:10.1029/2008JE003134.

Hashimoto, G. L., Sugita, S., Sep. 2003. On observing the compositional variability of the surface of Venus using nightside near-infrared thermal radiation. Journal of Geophysical Research (Planets) 108, $13-18$.

Haus, R., Arnold, G., Oct. 2010. Radiative transfer in the atmosphere of Venus and application to surface emissivity retrieval from VIRTIS/VEX measurements. Planetary and Space Science 58, 1578-1598.

Helbert, J., Müller, N., Kostama, P., Marinangeli, L., Piccioni, G., Drossart, P., Jun. 2008. Surface brightness variations seen by VIRTIS on Venus Express and implications for the evolution of the Lada Terra region, Venus. Geoph. Res. Let. 35, doi:10.1029/2008GL033609. 
Hide, R., Birch, N. T., Morrison, L. V., Shea, D. J., White, A. A., Jul. 1980. Atmospheric angular momentum fluctuations and changes in the length of the day. Nature 286, 114-117.

Howington-Kraus, E., Kirk, R., Galuszka, D., Hare, T., Redding, B., Mar. 2002. Validation of the USGS Magellan Sensor Model for Topographic Mapping of Venus. In: Lunar and Planetary Institute Conference Abstracts. Vol. 33 of Lunar and Planetary Inst. Technical Report. p. abstract no. 1986.

Ignatiev, N. I., Titov, D. V., Piccioni, G., Drossart, P., Markiewicz, W. J., Cottini, V., Roatsch, T., Almeida, M., Manoel, N., Aug. 2009. Altimetry of the Venus cloud tops from the Venus Express observations. Journal of Geophysical Research (Planets) 114, doi:10.1029/2008JE003320.

Konopliv, A. S., Banerdt, W. B., Sjogren, W. L., May 1999. Venus Gravity: 180th Degree and Order Model. Icarus 139, 3-18.

Lebonnois, S., Hourdin, F., Eymet, V., Crespin, A., Fournier, R., Forget, F., Jun. 2010. Superrotation of Venus' atmosphere analyzed with a full general circulation model. Journal of Geophysical Research (Planets) 115, doi:10.1029/2009JE003458.

Lecacheux, J., Drossart, P., Laques, P., Deladerriere, F., Colas, F., Jul. 1993. Detection of the surface of Venus at 1.0 micrometer from ground-based observations. Planetary and Space Science 41, 543-549.

Margot, J., Campbell, D., Jurgens, R., M.A., S., Feb. 2006. Earth-based Radar Measurements of Venus Length of day Variations. In: Chapman Conference on Venus as a Terrestrial Planet, Key Largo, Florida, USA.

Moroz, V. I., Mar. 2002. Estimates of visibility of the surface of Venus from descent probes and balloons. Planetary and Space Science 50, 287-297.

Mueller, N., Helbert, J., Hashimoto, G. L., Tsang, C. C. C., Erard, S., Piccioni, G., Drossart, P., Dec. 2008. Venus surface thermal emission at $1 \mu \mathrm{m}$ in VIRTIS imaging observations: Evidence for variation of crust and mantle differentiation conditions. Journal of Geophysical Research (Planets) 113, doi:10.1029/2008JE003118.

Parish, H. F., Schubert, G., Covey, C., Walterscheid, R. L., Grossman, A., Lebonnois, S., Mar. 2011. Decadal variations in a Venus general circulation model. Icarus 212, 42-65.

Pettengill, G. H., Briscoe, H. W., Evans, J. V., Gehrels, E., Hyde, G. M., Kraft, L. G., Price, R., Smith, W. B., May 1962. A radar investigation of Venus. Astronomical Journal 67, 181-190.

Pettengill, G. H., Ford, P. G., Johnson, W. T. K., Raney, R. K., Soderblom, L. A., Apr. 1991. Magellan - Radar performance and data products. Science 252, 260-265.

Pettengill, G. H., Ford, P. G., Wilt, R. J., Aug. 1992. Venus surface radiothermal emission as observed by Magellan. Journal of Geophysical Research 97, 13091-13102.

Press, W., Teukolsky, S., Vetterling, W., Flannery, B., 1992. Numerical Recipes in C, 2nd Edition. Cambridge University Press, Cambridge, UK.

Ragent, B., Esposito, L. W., Tomasko, M. G., Marov, M. I., Shari, V. P., 1985. Particulate matter in the Venus atmosphere. Advances in Space Research 5, 85-115.

Rappaport, N. J., Konopliv, A. S., Kucinskas, A. B., Ford, P. G., May 1999. An Improved 360 Degree and Order Model of Venus Topography. Icarus 139, 19-31.

Schubert, G., 1983. General circulation and the dynamical state of the Venus atmosphere. Venus, pp. 
681-765.

Seidelmann, P. K., Abalakin, V. K., Bursa, M., Davies, M. E., de Bergh, C., Lieske, J. H., Oberst, J., Simon, J. L., Standish, E. M., Stooke, P., Thomas, P. C., Jan. 2002. Report of the IAU/IAG Working Group on Cartographic Coordinates and Rotational Elements of the Planets and Satellites: 2000. Celestial Mechanics and Dynamical Astronomy 82, 83-111.

Shapiro, I. I., Dec. 1967. Theory of the radar determination of planetary rotations. Astronomical Journal $72,1309-1323$.

Shapiro, I. I., Campbell, D. B., de Campli, W. M., Jun. 1979. Nonresonance rotation of Venus. Astrophysical Journal Letters 230, L123-L126.

Shapiro, I. I., Chandler, J. F., Campbell, D. B., Hine, A. A., Stacy, N. J. S., Oct. 1990. The spin vector of Venus. Astrophysical Journal 100, 1363-1368.

Slade, M. A., Zohar, S., Jurgens, R. F., Oct. 1990. Venus - Improved spin vector from Goldstone radar observations. Astrophysical Journal 100, 1369-1374.

Smrekar, S. E., Nov. 1994. Evidence for active hotspots on Venus from analysis of Magellan gravity data. Icarus $112,2-26$.

Smrekar, S. E., Stofan, E. R., Mueller, N., Treiman, A., Elkins-Tanton, L., Helbert, J., Piccioni, G., Drossart, P., Apr. 2010. Recent Hotspot Volcanism on Venus from VIRTIS Emissivity Data. Science $328,605-608$.

Stofan, E. R., Smrekar, S. E., Bindschadler, D. L., Senske, D. A., Nov. 1995. Large topographic rises on Venus: Implications for mantle upwelling. Journal of Geophysical Research 100, 23317-23328.

Svedhem, H., Titov, D. V., McCoy, D., Lebreton, J.-P., Barabash, S., Bertaux, J.-L., Drossart, P., Formisano, V., Häusler, B., Korablev, O., Markiewicz, W. J., Nevejans, D., Pätzold, M., Piccioni, G., Zhang, T. L., Taylor, F. W., Lellouch, E., Koschny, D., Witasse, O., Eggel, H., Warhaut, M., Accomazzo, A., Rodriguez-Canabal, J., Fabrega, J., Schirmann, T., Clochet, A., Coradini, M., Oct. 2007. Venus Express - The first European mission to Venus. Planetary and Space Science 55, 16361652.

Titov, D. V., Svedhem, H., Koschny, D., Hoofs, R., Barabash, S., Bertaux, J.-L., Drossart, P., Formisano, V., Häusler, B., Korablev, O., Markiewicz, W. J., Nevejans, D., Pätzold, M., Piccioni, G., Zhang, T. L., Merritt, D., Witasse, O., Zender, J., Accomazzo, A., Sweeney, M., Trillard, D., Janvier, M., Clochet, A., Nov. 2006. Venus Express science planning. Planetary and Space Science 54, 1279-1297. Victor, W. K., Stevens, R., Jul. 1961. Exploration of Venus by Radar. Science 134, 46-48.

Wood, J. A., 1997. Rock Weathering on the Surface of Venus. In: Bougher, S. W., Hunten, D. M., Philips, R. J. (Eds.), Venus II: Geology, Geophysics, Atmosphere, and Solar Wind Environment. pp. $637-661$. 

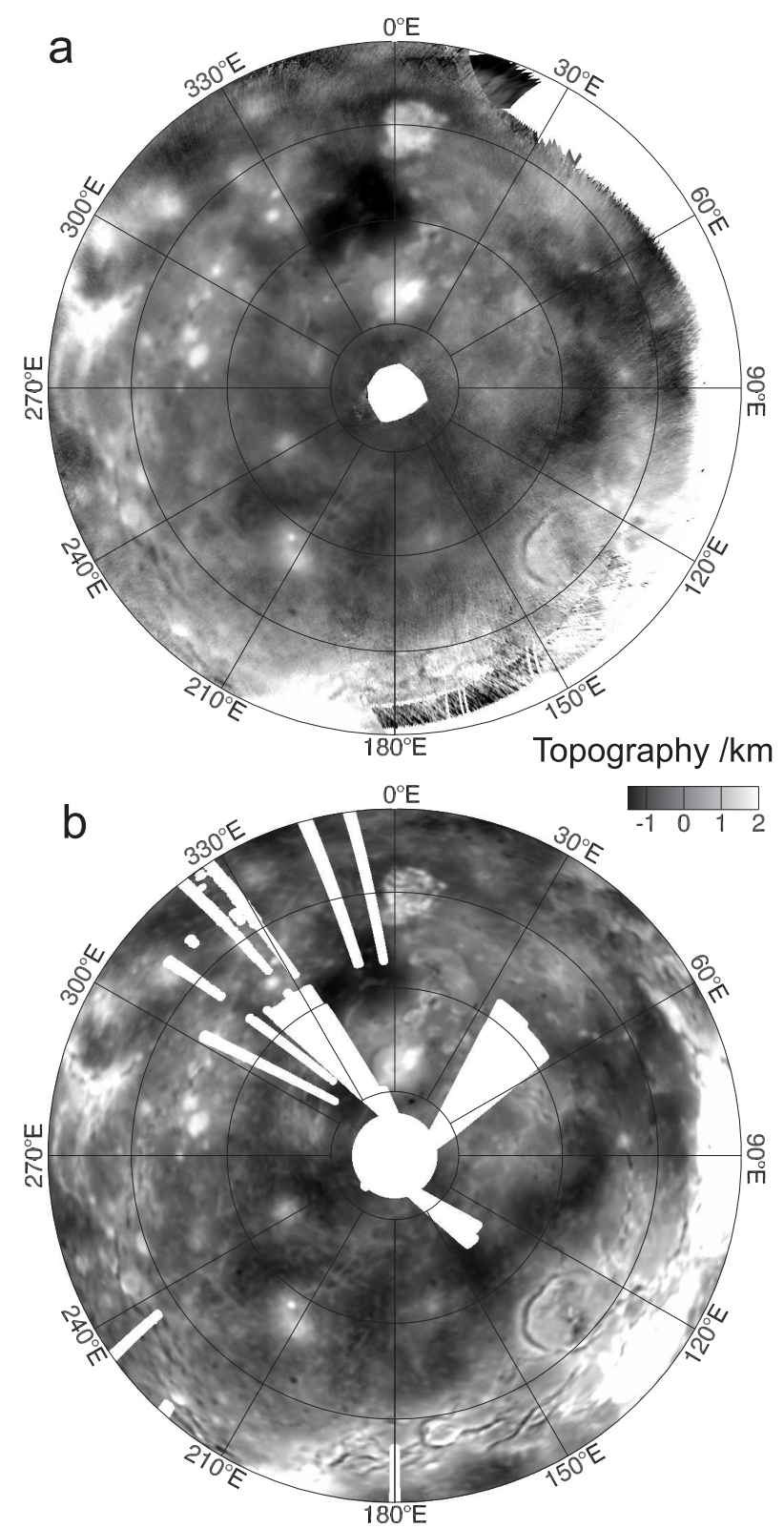

Figure 1: Topography maps of the southern hemisphere of Venus in Lamberts azimuthal equal area projection. a) derived from VIRTIS near infrared thermal emission data. b) Magellan altimetry (Rappaport et al., 1999) smoothed to resemble thermal emission resolution. Areas within $100 \mathrm{~km}$ distance of missing data are left blank. 

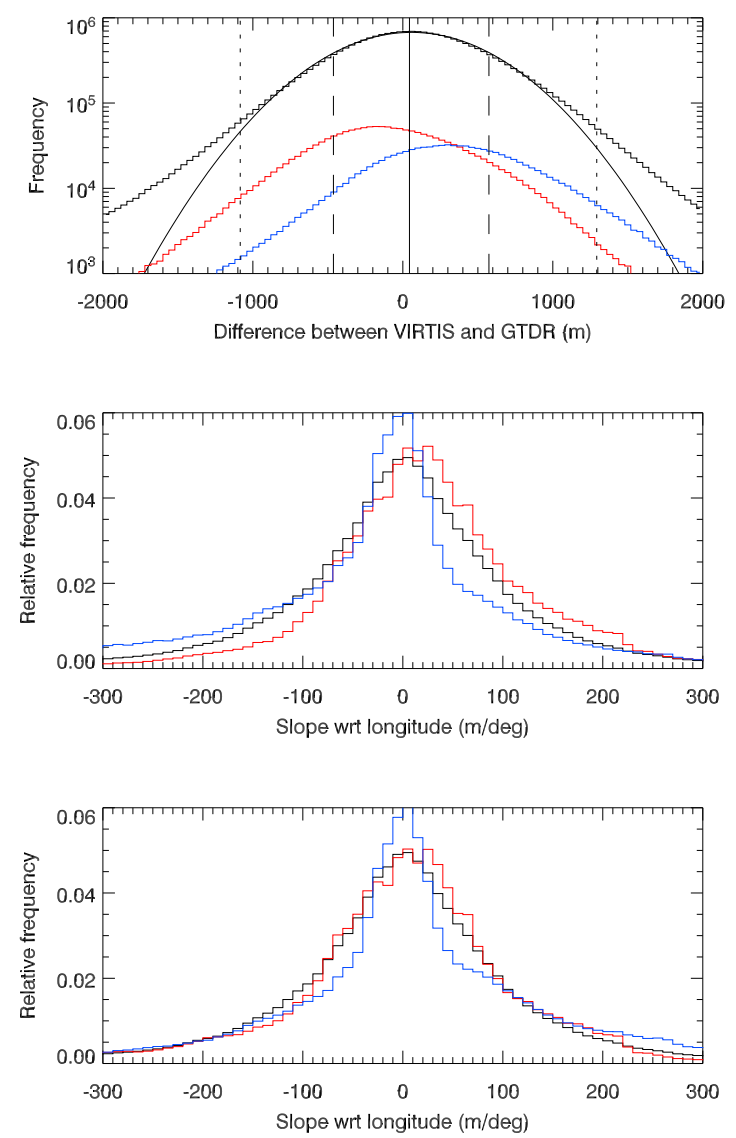

Figure 2: a) Frequency distribution of differences between VIRTIS data and GTDR. The black histogram represents the whole data set, the solid graph is a fit of a gaussian with center at $58 \mathrm{~m}$ and standard deviation of $494 \mathrm{~m}$. The solid vertical line represents the median deviation at $44 \mathrm{~m}$ while the long-dashed represent the 16 th and 84 th percentile -i.e. $1 \sigma$ in a normal distribution- and the short dashed the 2.3th and 97.7th percentile. The red and blue histograms represent subsets of the data with a local bias of less than $-300 \mathrm{~m}$ and more than $300 \mathrm{~m}$, respectively. b) The relative frequency distributions of slopes with respect to longitude for the whole set and the two subsets. c) A reselection of the outlying subsets correcting for an assumed offset in longitude of $-0.3 \mathrm{deg}$. 


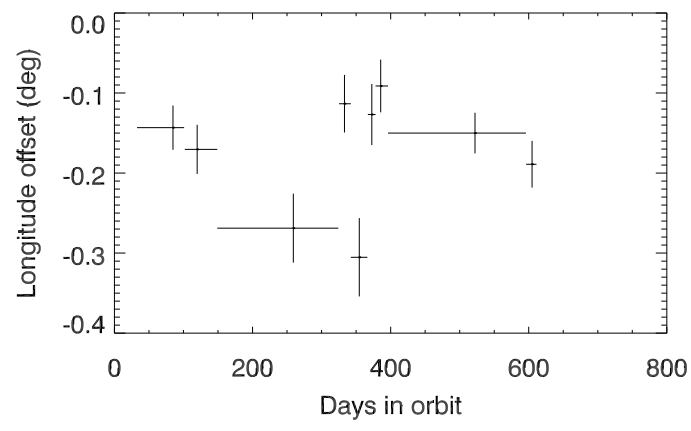

Figure 3: Offset derived from similarly sized subsets created by assigning images in order of data acquisition time. Horizontal bars denote period of data acquisition for each subset. The varying data acquisition duration of subsets is due to the varying rate of data produced by VIRTIS. Venus Express (VEX) orbit insertion was on 4 April 2006, 5577 Julian days after the median Magellan data acquisition time. The $\chi^{2}$ error estimates are to small for the scale of this plot. The vertical error bars correspond to the confidence interval derived from the 'bootstrap' method.

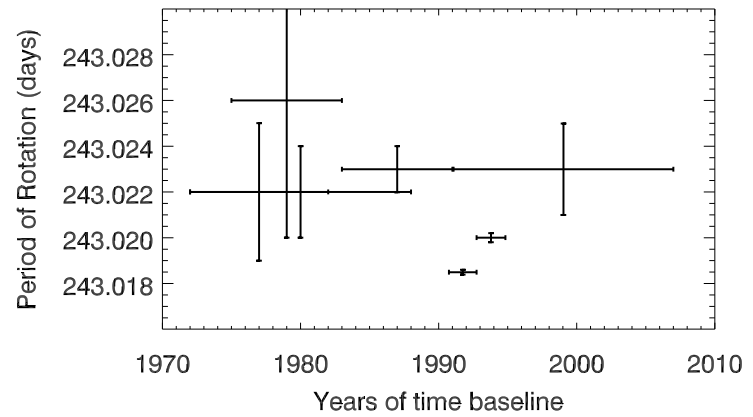

Figure 4: The most recent estimates of the period of rotation and the time baseline of measurements. The full models and their sources are given in table 3. The horizontal bars show the period over which the data for each estimate was acquired. 